Bull. Mater. Sci., Vol. 17, No. 6, November 1994, pp. 1119-1129. (C) Printed in India.

\title{
Electroclinic measurement of the coefficients of Landau expansion of some ferroelectric liquid crystals ${ }^{\dagger}$
}

\author{
H P PADMINI*, N V MADHUSUDANA and B SHIVKUMAR \\ Raman Research Institute, Bangalore 560 080, India \\ *Present address: The National College, Basavanagudi, Bangalore 560004 , India
}

\begin{abstract}
The electroclinic effect in the smectic A phase of ferroelectric liquid crystals is a sensitive probe for measuring the soft mode response near the A-C* transition point. It is possible to evaluate several coefficients of the Landau theory describing the $\mathrm{A}-\mathrm{C}$ * transition by simultaneously measuring the frequency dependence of both the optical signal and the current through the sample. We have made such measurements on a couple of homologous series $[2 S, 3 S]-4^{\prime}-(2$-chloro-3-methyl pentanoyloxy) phenyl-trans-4"-n alkoxy cinnamates synthesized in our laboratory. The results show that the Landau meanfield theory is adequate to describe the $\mathrm{A}-\mathrm{C}^{*}$ phase transition in these compounds.
\end{abstract}

Keywords. Electroclinic effect; ferroelectric liquid crystals; soft mode.

\section{Introduction}

The smectic $\mathrm{C}^{*}$ liquid crystal is composed of chiral molecules. It consists of a stack of fluid layers in which the molecular long axes are tilted with respect to the layer normal. Meyer et al (1975) showed that the symmetry of such a medium allows it to be a ferroelectric. Consider a vertical cross section of a smectic $C^{*}$ layer passing through the layer normal $\mathrm{Z}$ and the director $\hat{n}$ (figure 1 ). The structure has a two-fold rotation axis about $X$. As the molecules are chiral, the $Y Z$ plane is not a mirror plane. The two-fold axis sustains the properties described by a polar vector along that axis. When the molecules have permanent transverse dipole moments, there can be a polarization $P$ along the two-fold axis, i.e. the medium can become ferroelectric. The polarization is normal to the plane containing the director and the layer normal and hence such a ferroelectric is called transverse ferroelectric.

Many compounds exhibit a second order smectic $C^{*}$ to smectic A transition. In the latter, the molecules are upright and because of the rotational symmetry about the layer normal, it is not a ferroelectric liquid crystal. However, as was shown by Garoff and Meyer $(1977,1979)$, an electric field applied in the plane of the layers can produce a tilt of the molecules in the orthogonal plane of such a smectic A liquid crystal. This is known as the electroclinic effect. The electric field $E$ couples to the transverse component of the molecular permanent electric dipole $(p)$. This biases the free rotation of the molecules about their long axes since $p$ tends to be parallel to the applied field. The system has a two-fold axis along the electric field. The plane containing the layer normal and $p$ is a mirror plane in a non-chiral system. In the chiral system this mirror plane is lost. A molecular tilt with respect

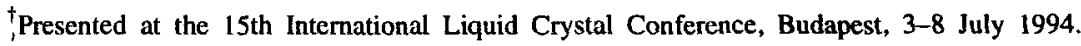




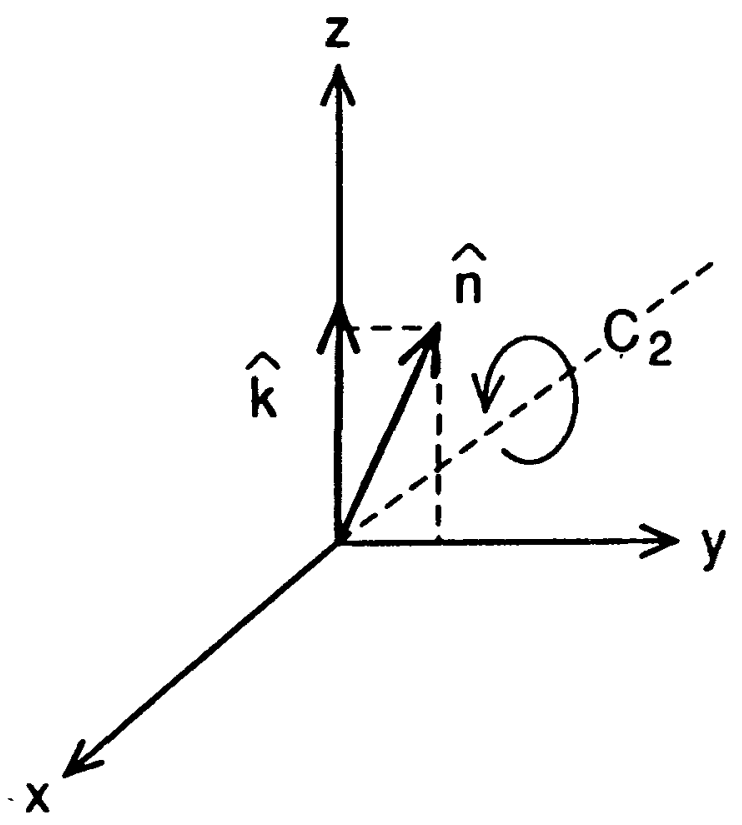

Figure 1. The $C_{2}$ symmetry operation in a ferroelectric smectic layer.

to the layer normal can then be induced in the orthogonal plane. This tilt is a linear function of the field (for small fields). A change in the director orientation produces a change in the direction of the optic axis, which gives rise to a linear electrooptic response. The electroclinic effect resembles piezo electricity in crystalline phases in some respects. However the fluid nature of the liquid crystalline phase does not allow any static shear strains which are associated with piezoelectricity in solid crystals. In the lowest order, the mean field expression for the free energy density of a smectic A liquid crystal made up of chiral molecules is given by (Garoff and Meyer 1979)

$$
F=F_{0}+\frac{1}{2} A(T) \theta^{2}+\frac{1}{2} \chi_{p}^{-1} P^{2}-P E-\frac{\varepsilon_{-} E^{2}}{2}-c P \theta
$$

where $F_{0}$ is the ground state free energy of the smectic A phase, $A(T)$ the temperature dependent Landau coefficient given by $a\left(T-T_{c}\right), \theta$ the induced tilt angle, $\chi_{p}$ the generalized susceptibility, $P$ the induced polarization, $E$ the external field, $c$ the electroclinic coupling constant and $\varepsilon_{\infty}$ the high frequency dielectric constant. Minimizing the free energy with respect to $\theta$ and $P$, we get

and

$$
\theta=\frac{\chi_{p} c E}{A\left(T^{*}\right)},
$$

$$
P=\chi_{p} E+\frac{\chi_{p}^{2} c^{2}}{A\left(T^{*}\right)} E
$$


where

$$
\begin{aligned}
A\left(T^{*}\right) & =a\left(T-T_{c}^{*}\right), \\
T_{c}^{*} & =T_{c}+\frac{\chi_{p} c^{2}}{a} .
\end{aligned}
$$

Due to the linear coupling $c P \theta$, the transition temperature is displaced in a chiral compound compared to its non-chiral analogue. When a sinusoidal field $E e^{i \omega t}$ is applied to the cell, $A\left(T^{*}\right)$ is to be replaced by $\left[A\left(T^{*}\right)\right]+i$ w where $\eta$ is the soft-mode viscosity. Then we can expect the frequency dependences of the electroclinic tilt and of the electroclinic contribution to $P$ to be Lorentzians with a relaxation frequency proportional to $\left[\tilde{a}\left(\left(T-T_{c}^{*}\right) / T_{c}^{*}\right)\right]$, where

$$
\tilde{a}=a T_{c}^{*}
$$

The phenomenological equation of motion can be written as

i.e.

$$
\eta(i \omega \theta)+\tilde{a}\left(\frac{T-T_{c}^{*}}{T_{c}^{*}}\right) \theta=c \chi_{p} E
$$

$$
\theta=\frac{c \chi_{p} E}{\tilde{a}\left(\frac{T-T_{c}^{*}}{T_{c}^{*}}\right)(1+i \omega \tau)},
$$

where

$$
\tau=\frac{\eta}{\tilde{a}\left(\frac{T-T_{c}^{*}}{T_{c}^{*}}\right)},
$$

is the relaxation time of the fluctuations of the order parameter $\theta$. These equations can be rewritten in forms which will be useful to estimate the coefficients of the Landau expression following the procedure of Dupont et al (1991). Using (7), the RMS value of $\theta$ can be written as

$$
|\theta|=\frac{c \chi_{p} E}{\tilde{a}\left(\frac{T-T_{c}^{*}}{T_{c}^{*}}\right)} \frac{1}{\sqrt{1+\omega^{2} \tau^{2}}}
$$

When the frequency is very low such that $\omega \tau \ll 1$,

$$
e=\frac{\theta}{E} \approx \frac{c \chi_{p}}{\tilde{a}\left(\frac{T-T_{c}^{*}}{T_{c}^{*}}\right),}
$$


and using (3),

$$
P=E\left[\chi_{p}+\frac{c^{2} \chi_{p}^{2}}{\tilde{a}\left(\frac{T-T_{c}^{*}}{T_{c}^{*}}\right)}\right]
$$

The dielectric permittivity of the medium along - a direction perpendicular to the director is then given by

$$
\varepsilon_{\perp}=\left(\varepsilon_{o}+\chi_{p}\right)+\frac{c^{2} \chi_{p}^{2}}{\tilde{a}\left(\frac{T-T_{c}^{*}}{T_{c}^{*}}\right)}
$$

In the above equation, we have also to add the contribution from the very high frequency dielectric constant which arises from the polarizability due to the electron cloud. This contribution $\simeq \varepsilon_{0} \chi_{\infty}$. where $\chi_{0}=n_{o}^{2}$. Here $n_{o}$ is the ordinary refractive index.

Using (10) and (12), by plotting $\varepsilon_{\perp}$ vs $e$, the $Y$ intercept yields $\left(\varepsilon_{o}+\varepsilon_{o} \chi_{\infty}+\chi_{p}\right)$ and the slope gives $c \chi_{p}$.

\section{Experimental results and discussion}

A block diagram of the experimental arrangement is shown in figure 2 . Two conductive indium tin oxide coated glass plates are treated with polyimide and unidirectionally rubbed. A cell with a typical thickness of 7 to $10 \mu$ is made using these plates. It is filled with the ferroelectric sample in the isotropic phase and is mounted in a cell holder, placed in an INSTEC HSI-i microscope hot stage. The

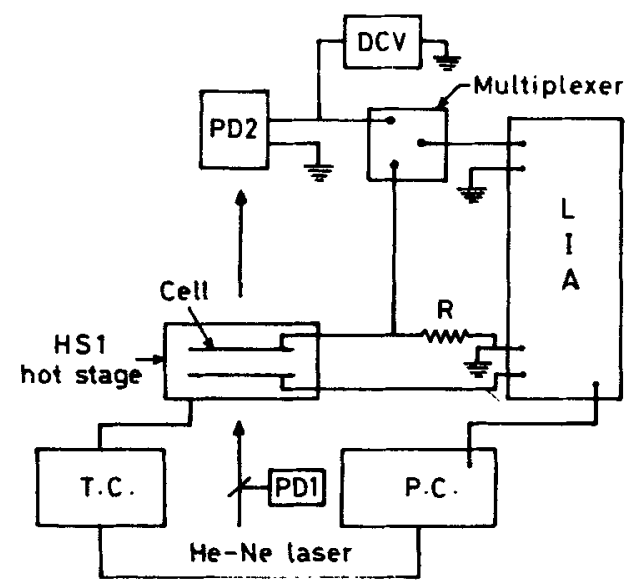

Figure 2. Block diagram of the experimental set-up used to measure the current and electrooptic response. 
temperature of the cell is regulated with the help of a PC. The temperature stability is around $10 \mathrm{mK}$. A standard resistance $R=10 \mathrm{ohms}$ is connected in series with the cell to monitor the current through the cell. A He-Ne laser is used as the light source. Using a glass plate as a partial reflector, the laser intensity is monitored using a photodiode (OSI-5K). The intensity of light transmitted through the cell is measured using a PIN photodiode (Hamamatsu 1722) which has a response time of $5 \mathrm{~ns}$. The output of the photodiode is connected to a preamplifier which is built around the fast operational amplifier BB3551. The preamplified output is fed to a lock-in-amplifier (PAR 5301) for optical signal measurement at the frequency of the applied field, and also to a DC multimeter (HP3457 A) to measure the DC part of the signal. We used a relay operated multiplexer as a switching device between the optical and conductivity signals. Suitable programmes were used to measure both the signals under identical conditions of frequency, applied voltage and temperature of the sample (spectrum interface).

We conducted measurements on two compounds of the homologous series [2S,3S]4'-(2-chloro-3-methylpentanoyloxy) phenyl trans-4"-n-alkoxycinnamates synthesized in our chemistry laboratory (Shivkumar et al 1991). The general structural formula is shown in figure 3 and the transition temperatures are given in table 1.

The samples had some ionic impurities, which make a considerable contribution to the conductivity signal at low frequencies. But at higher frequencies $(\sim \mathrm{KHz})$ ions cannot follow the field and the contribution due to the capacitative coupling becomes prominent. This allows us to measure the electroclinic contribution to the dielectric constant which is needed in the analysis.

We have measured the frequency dependence of the electroclinic $f$ signal starting from a few degrees above the A-C* transition temperature up to $T_{\mathrm{AC}}$ for $\mathrm{D} 9$ and D10. The data was fitted to a Lorentzian $\theta(\omega)$ (see (9)) using a nonlinear least squares fitting programme. The calculated variations are shown as lines in figure 4 for D9. The relaxation frequency $\tau^{-1}$ was obtained as a function of temperature using this procedure (figure 5). It shows that the relaxation frequency increases linearly with temperature in accordance with the prediction of the Landau theory (see (8)). The electroclinic contribution to the dielectric constant relaxes beyond that frequency. Similar measurements were made on D10 also. Figure 6 shows the $\tau^{-1}$ variation with temperature for D10.

The typical frequency dependence of the current (which is essentially capacitative)

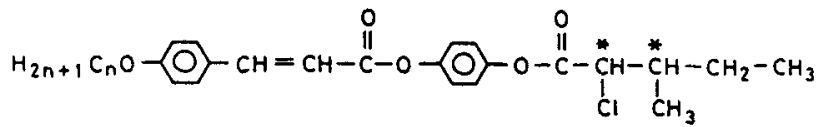

Figure 3. The gencral structural formula of $[2 S, 3 S]-4^{\prime}-(2$-chloro-3-methyl pentanoyloxy) phenyl-truns-4"-n-alkoxy cinnamates used in our experiments.

Table 1. The transition temperatures (in ${ }^{\circ} \mathrm{C}$ ) of [2S,3S]-4'-(2-chloro-3-methyl-pentanoyloxy)phenyl trans- 4"-n-alkoxycinnamates.

\begin{tabular}{|c|c|c|c|c|c|c|c|c|c|c|}
\hline Compound & $n$ & $\mathrm{C}$ & & $S_{C^{*}}$ & & $S_{\mathrm{A}}$ & & $N^{*}$ & & $I$ \\
\hline D9 & 9 & . & 69.0 & . & $78-0$ & . & 95.0 & . & 97.0 & \\
\hline DI0 & 10 & . & $56 \cdot 0$ & . & $80 \cdot 0$ & . & 98.5 & . & 99.0 & \\
\hline
\end{tabular}



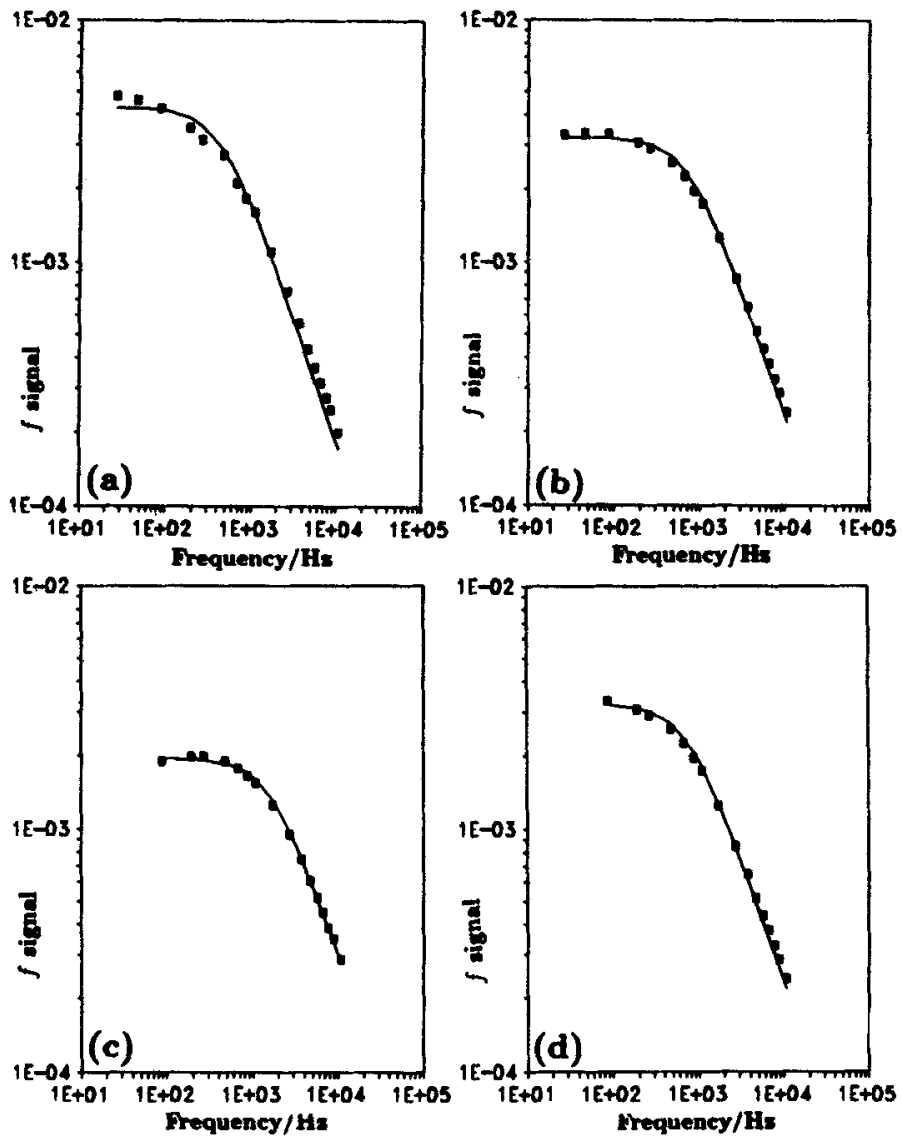

Figure 4. Variation of $f$ signal as a function of frequency for the compound D9 at (a) $339.3 \mathrm{~K}$, (b) $3.39 .5 \mathrm{~K}$. (c) $339.7 \mathrm{~K}$ and (d) $340.1 \mathrm{~K}$. Solid lines show the fitted Lorentzians.

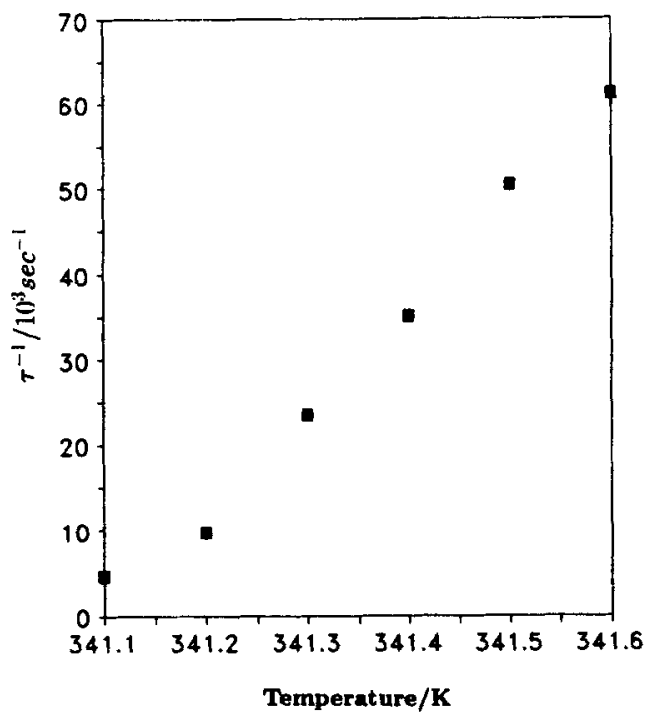

Figure 5. Variation of $\tau^{-1}$ as a function of temperature for $D 9$. 


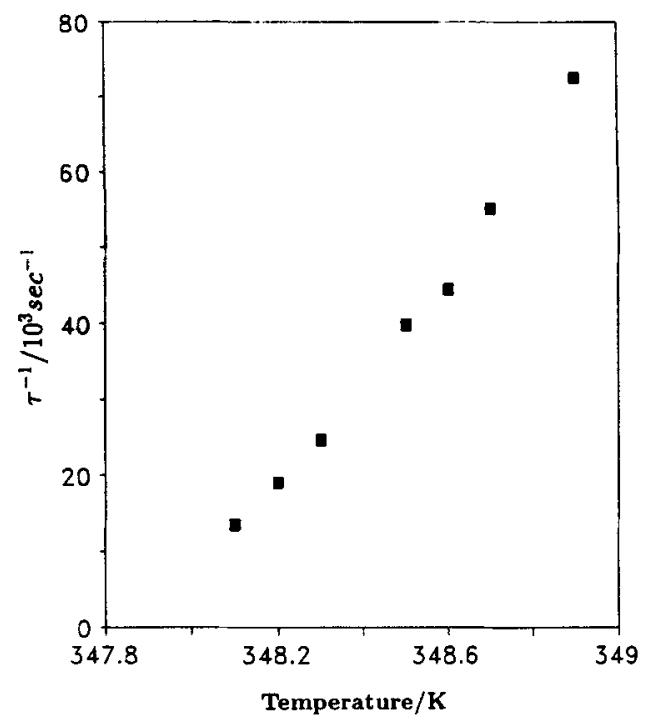

Figure 6. Variation of $\tau^{-1}$ as a function of temperature for DIO.

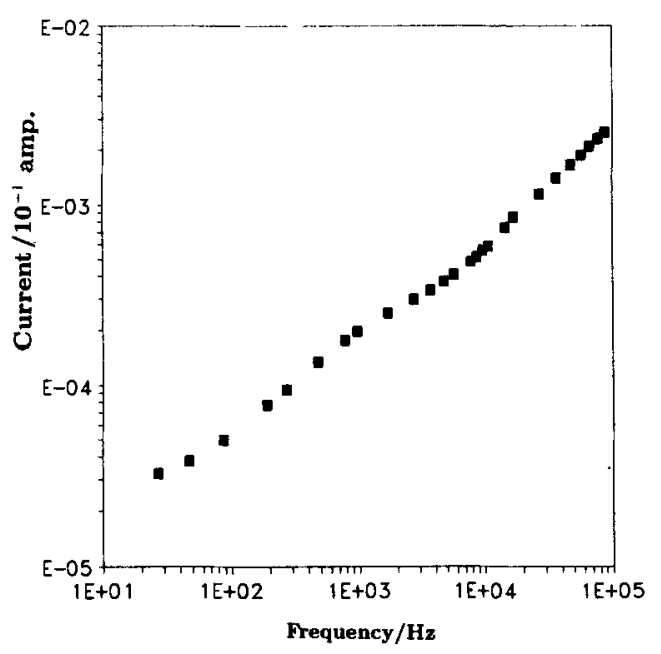

Figure 7. Variation of current as a function of frequency for the compound D9 at $342 \mathrm{~K}$.

is shown in figure 7 . It shows that there is a change of slope when the electroclinic contribution to the current relaxes.

For D9 and D10, we have made measurements of the current as a function of temperature at 1960 and $5700 \mathrm{~Hz}$ respectively, simultaneously with the measurement of the optical signal. We use the data in a temperature range in which these frequencies are below the relaxation frequency and use $(10-12)$ in further analysis of the data. 


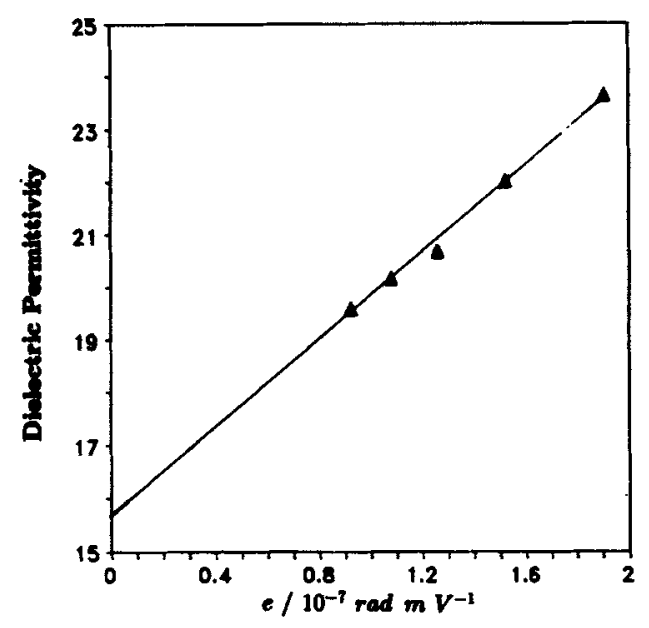

Figure 8. Dielectric permittivity as a function of the EC coefficient for the compound D9

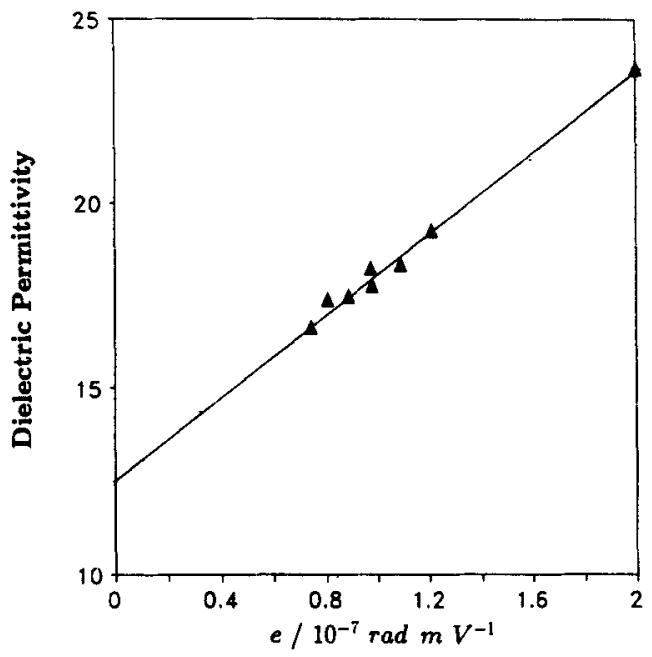

Figure 9. Dielectric permittivity as a function of the EC coefficient for the compound D10.

The capacitance of the cell is given by

$$
C=\left|\frac{l}{\omega V}\right|=\varepsilon_{\perp} C_{0}+C_{\text {spacer }},
$$

where $\varepsilon_{\perp}$ is the dielectric permittivity of the medium along a direction perpendicular to the director, $C_{0}$ the capacitance of the part of the empty cell without the spacer which was calculated by the geometrical parameters of the cell, $C_{\text {spacer }}$ is the capacitance of the part of the cell which had mylar spaces which was also calculated 


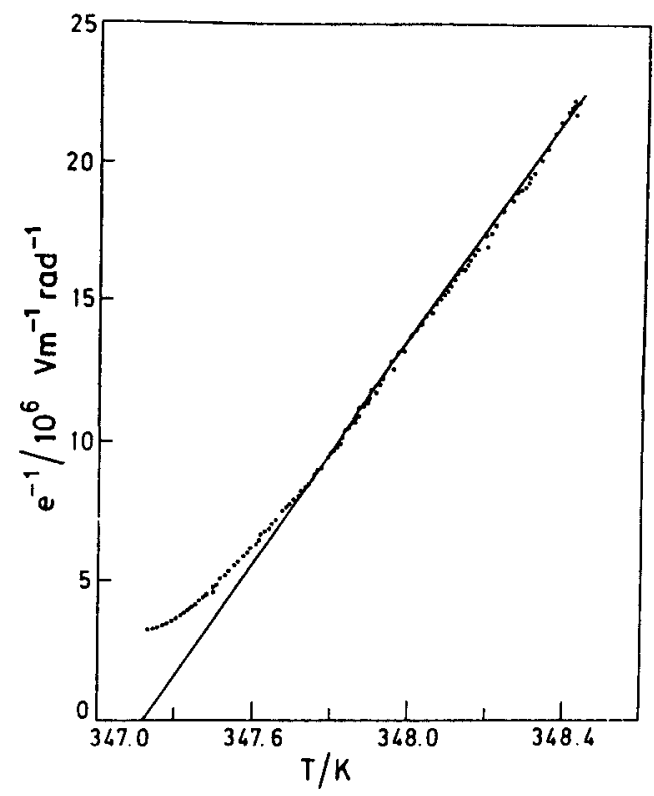

Figure 10. Temperature dependence of the inverse electroclinic coefficient, $e^{-1}$ of D9 at $1960 \mathrm{~Hz}$.

by the geometrical parameters. The above equation can be rewritten as

$$
\frac{C}{C_{0}}=\frac{C_{\text {spacer }}}{C_{0}}+\varepsilon_{1},
$$

from which $\varepsilon_{\perp}$ was evaluated. We plotted $\varepsilon_{\perp} / \varepsilon_{0}$ vs $e=(\theta / E)$ at various temperatures. Figure 8 shows the variation for $D 9$ and figure 9 for D10.

As expected from (10) to (12) the variation of $\varepsilon_{1}$ vs $e$ is a straight line within the accuracy of our measurements. As discussed after (12) the Y-intercept yields $\left[1+\chi_{\infty}+\left(\chi_{p} / \varepsilon_{o}\right)\right]$ and the slope gives $c \chi_{p}$. Here $\chi_{\infty}=n_{o}^{2}$, where $n_{o}$ is the ordinary index $\simeq 1.6$. Hence from the $Y$ intercept we can calculate $\chi_{p}$ and using this value the coupling coefficient $c$ can be calculated from the slope. From the graph of $e^{-1}$ vs $T$, using (10), the corresponding slope gives $\tilde{a}$. In the case of D9 and D10, from figures 10 and 11 the slopes have the magnitudes $2.1 \times 10^{7}$ and $1.6 \times 10^{7}$ respectively. Using (8), by substituting the value of $\tilde{a}$, the viscosity coefficient $\eta$ can be c 2alculated. We have shown the calculated values of the various quantities in table 2.

The values given in table 2 are broadly in the expected range, and comparable to those given by Dupont et al (1991) for other compounds. Our values of $\chi_{p} / \varepsilon_{o}$ for D9 and D10 are somewhat larger than the values obtained by Dupont et al (1991) for their compounds. This may partly be due to the presence of an additional $\mathrm{COO}$ group in the molecular structure of the compounds that we have used. This will enhance the lateral dipole moment of the molecule. The viscosity coefficient $\eta$ is $0.01 \mathrm{Ns} / \mathrm{m}^{2}$ which is of the right order for liquid crystals at high temperatures. These numbers are also consistent with the fitting parameters obtained on another compound D8 which belong to the same homologous series (Padmini 


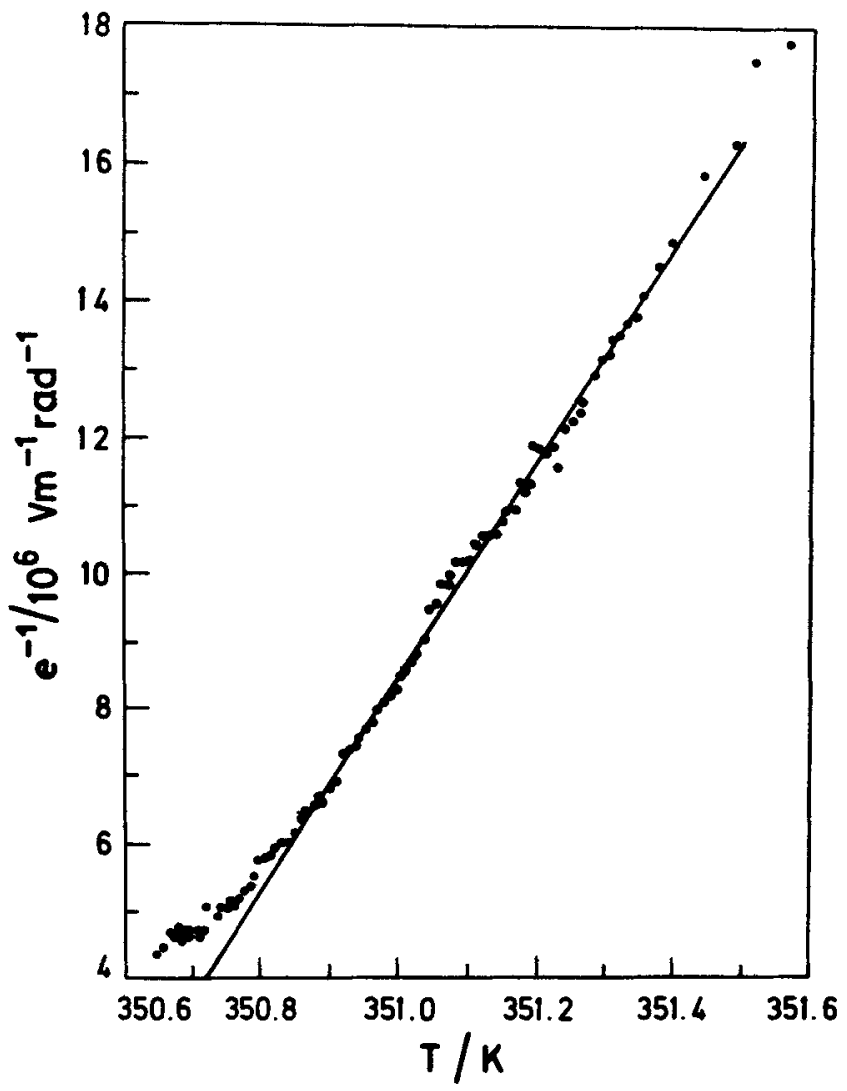

Figure 11. Temperature dependence of the inverse electroclinic cocfificient, $e^{-1}$ of D10 at $1960 \mathrm{~Hz}$.

Table 2. Coefficients of the Landau theory.

\begin{tabular}{lcc}
\hline & \multicolumn{2}{c}{ Compound } \\
\cline { 2 - 3 } Coefficients & $\mathrm{D} 9$ & $\mathrm{D} 10$ \\
\hline$\chi_{p} / \varepsilon_{o}$ & 11.6 & 8.5 \\
$\chi_{p}\left(\mathrm{~cm}^{-2} \mathrm{rad}^{-1}\right)$ & $3.73 \times 10^{-4}$ & $4.87 \times 10^{-4}$ \\
$c\left(\mathrm{NC}^{-1} \mathrm{rad}^{-1}\right)$ & $0.36 \times 10^{7}$ & $0.65 \times 10^{7}$ \\
$a\left(\mathrm{Nm}^{-2} \times \mathrm{K}^{-1}\right)$ & $0.78 \times 10^{4}$ & $0.78 \times 10^{4}$ \\
$\Delta T_{c}(\mathrm{~K})$ & 1.7 & 0.4 \\
$\eta\left(\mathrm{N} \mathrm{sm}^{-2}\right)$ & 0.01 & 0.01 \\
\hline
\end{tabular}

et al .1993) where we analyzed only the optical data in terms of the Garoff-Meyer model. However, we should note that in the present analysis $\eta$ is assumed to be temperature independent.

Thus the dynamic measurements on the soft mode electroclinic effect in the $A^{*}$ phase can be used to estimate all the parameters of the linearized Landau model of the A-C* transition. 


\section{Acknowledgement}

We thank Ms S Sarala for helping us in carrying out the experiments.

\section{References}

Dupont L, Glogarova M, Marcerou J P, Nguyen H T, Destrade C and Lejcek L 199/ J. Phys // 1831 Garoff S and Meyer R B 1977 Phys. Rev. Lett. 38848

Garoff S and Meyer R B 1979 Phys. Rev. A19 338

Meyer R B, Liebert L, Strzelecki L and Keller P 1975 J. Phys. Lett. 64307

Padmini H P, Pratibha R, Madhusudana N V and Shivkumar B 1993 Liquid Crystals 14435

Shivkumar B, Sadashiva B K. Krishna Prasad S and Khened S M 1991 Ferro electrics 114273 